\title{
ON ESTIMATING NUMBER OF GENES BY GENOTYPE ASSAY
}

\author{
W. G. HILL and P. J. AVERY \\ Institute of Animal Genetics, West Mains Rood, Edinburgh EH9 3JN
}

Received 8.ix.77

\begin{abstract}
Summary
The method of genotype assay, proposed by Jinks and Towey (1976) for estimating the number of effective factors in a polygenic system, assumes independent segregation if applied to number of genes. Their results are extended to include the case of linked genes, and the ratio of expected number of effective factors to number of genes is computed for a range of models. Unless all genes are on different chromosomes or many generations of inbreeding are used, the estimate of gene number is biased downwards.
\end{abstract}

\section{INTRODUCTION}

JINKS AND TOWEY (1976), in their paper " Estimating the number of genes in a polygenic system", proposed a method for estimating the number of effective factors (Mather and Jinks, 1971) responsible for variation of a continuous trait. In genotype assay of the $\mathrm{F}_{n}$ generation derived by continuous selfing from a cross between two pure breeding lines, the proportion of individuals that are heterozygous at one or more loci is determined by a progeny test using their $\mathrm{F}_{n+2}$ grand-progeny families. The observed proportion of heterozygous $\mathrm{F}_{n}$ individuals is then equated to a theoretical expectation which is a function of the number of genes involved, if they are all unlinked, or the number of effective factors.

The calculations used in genotype assay estimate the number of independantly segregating blocks of genes, i.e. of effective factors. If there is no linkage (no more than one gene per block) effective factors and genes are the same. If there is linkage, there are more genes than effective factors. Furthermore, the blocks of linked genes are broken up by recombination so that the number of effective factors estimated in successive generations may increase. In this note we investigate how estimates of effective factors from genotype assay are expected to correspond to the actual number of genes at which the two parents of the $F_{1}$ differ, using the standard genetic model of genes linked on chromosomes.

\section{ANALYSIS}

Jinks and Towey consider two estimates, an upper estimate $\mathrm{P}_{\max }$ in which no loci are assumed to be completely dominant or to have equal effect, so that any segregation can be detected, and a lower estimate $\mathrm{P}_{\min }$ in which all loci are completely dominant and have equal effect (relational and internal balance). We shall consider how such $\mathrm{P}$ values change as a result of linkage and initial coupling or repulsion. Results will be given in detail for the effects on $P_{\max }$, and then outlined for others. 
(i) No complete dominance, all effects unequal (no internal or relational balance)

Independent genes. The probability that a randomly sampled individual of generation $n$ of selfing is heterozygous at a locus which is heterozygous in the $F_{1}$ is $1 / 2^{n-1}$; the probability that two randomly chosen progeny of a heterozygote will differ in genotype at this locus is $5 / 8$ and hence the probability that these progeny differ at this locus is $5 / 2^{n+2}$. If the $F_{1}$ was heterozygous at $k$ independent loci, the probability that the progeny differ at one or more loci is thus Jinks and Towey's $P_{\max }$, since any segregation is detected,

$$
\mathrm{P}_{\max }=1-\left(1-5 / 2^{n+2}\right)^{k}
$$

(Jinks and Towey, 1976). Jinks and Towey give graphs of $\mathrm{P}_{\max }$ against $k$ for $n=2,3$ and 5 generations.

Linked gene pairs. Although the algebra is straightforward, it rapidly becomes unwieldy with increase in number of loci. Therefore we give exact results for two loci on each chromosome and resort to Monte Carlo simulation for more. Consider two loci, with a recombination fraction $c$ between them, and with the $\mathrm{F}_{1}$ being a double heterozygote, either coupling or repulsion. The probability, $H_{n}$, that a randomly sampled individual is a double heterozygote (coupling or repulsion) at generation $n$ is, from Jennings (1917),

$$
H_{n}=\frac{1}{2}\left[(1-c)^{2}+c^{2}\right] H_{n-1}=\left[\frac{1}{2}\left(1-2 c+2 c^{2}\right)\right]^{n-1},
$$

and the probability, $h_{n}$, that it is a single heterozygote is

$$
h_{n}=\frac{1}{2} h_{n-1}+2 c(1-c) H_{n-1}=\left[1-\left(1-2 c+2 c^{2}\right)^{n-1}\right] / 2^{n-2} .
$$

The probability, $Q_{D \mid H}$, that a pair of individuals chosen at random from the progeny of a selfed double heterozygote differ at one or more loci (assuming coupling and repulsion heterozygotes can not be distinguished) is

$$
Q_{D \mid H}=1-\frac{3}{8}\left[(1-c)^{4}+c^{4}+4 c^{2}(1-c)^{2}\right]
$$

and for single heterozygotes, $Q_{D \mid h}=5 / 8$. Hence the probability that a difference is detectable by progeny testing is obtained as

$$
\mathrm{P}_{n, c}=H_{n} Q_{D \mid H}+h_{n} Q_{D \mid h} .
$$

Values of $\mathrm{P}_{n, c}$ are given for a range of $n$ and $c$ values in table 1 . Values of $\mathbf{P}_{n, c}$ are, of course, less than $\mathbf{P}_{n, 0.5}$ which is Jinks and Towey's $\mathbf{P}_{\max }$ for two loci.

Several chromosomes. If there are $m$ chromosomes each with two loci having recombination fraction $c$ between them, the probability of detection, $\mathrm{P}_{\max }^{*}$ say, is

$$
\mathrm{P}_{\max }^{*}=1-\left(1-\mathrm{P}_{n, c}\right)^{m} .
$$

Equating (1) and (3) gives the expected prediction of the number of effective factors, given that there are, say, $g=2 m$ genes:

thus

$$
\left(1-\mathrm{P}_{n, c}\right)^{g / 2}=\left(1-5 / 2^{n+2}\right)^{k}
$$

$$
k=\left[g \log \left(1-\mathbf{P}_{n, c}\right)\right] /\left[2 \log \left(1-5 / 2^{n+2}\right)\right] .
$$


TABLE 1

Values of probability of detection $\left(P_{\mathrm{n}, \mathrm{c}}\right)$ for a pair of linked loci as a function of generation $(\mathrm{n})$ and recombination fraction (c), and corresponding ratio d of predicted number of effective factors to number of genes

\begin{tabular}{|c|c|c|c|c|c|c|c|c|c|}
\hline & \multicolumn{5}{|c|}{$\mathrm{P}_{n, c}$} & \multicolumn{4}{|c|}{$d \uparrow$} \\
\hline & 0.5 & $0 \cdot 2$ & 0.1 & 0.05 & 0.01 & 0.2 & $0 \cdot 1$ & 0.05 & 0.01 \\
\hline 1 & 0.8594 & $0 \cdot 8074$ & 0.7418 & 0.6912 & 0.6396 & 0.840 & 0.690 & 0.599 & 0.520 \\
\hline & 0.5273 & $0 \cdot 4745$ & 0.4166 & 0.3721 & 0.3259 & 0.859 & 0.719 & 0.621 & 0.526 \\
\hline & $0 \cdot 2881$ & $0 \cdot 2613$ & 0.2271 & $0 \cdot 1981$ & $0 \cdot 1659$ & 0.891 & 0.758 & 0.650 & 0.534 \\
\hline 5 & 0.0766 & 0.0722 & 0.0638 & 0.0547 & 0.0429 & 0.941 & 0.827 & 0.706 & 0.550 \\
\hline & 0.0097 & $0 \cdot 0095$ & 0.0088 & 0.0076 & 0.0056 & 0.978 & 0.900 & 0.779 & 0.576 \\
\hline
\end{tabular}

$\dagger$ For $c=0.5, d=1$ and for $c=0, d=0.5$ for all $n$.

The ratio $d=k / g$, the proportional underestimation of gene number, is independent of the number of chromosomes in this model. Values of $d$ are also given in table 1 .

Several loci. More generally, let $\mathrm{P}_{n, g_{i}, c_{i}}$ be the probability of detection for a chromosome having $g_{i}$ genes and vector $\mathbf{c}_{i}$ of recombination fractions, and let $g=\Sigma g_{i}$. Then

and

$$
\mathrm{P}_{\max }^{*}=1-\prod_{i=1}^{m}\left(1-\mathrm{P}_{n, g_{i}, c_{i}}\right)
$$

$$
d=\sum_{i=1}^{m} \log \left(1-\mathrm{P}_{n, g_{i}, c_{i}}\right) /\left[g \log \left(1-5 / 2^{n+2}\right)\right] .
$$

Some examples of $\mathrm{P}_{n, g_{i}, c_{i}}$ obtained by Monte Carlo simulation are given in table 2 , together with corresponding values of $d$ if all chromosomes have the same number and distribution of genes. The values of $d$ are, of course, further from unity than with only two genes per chromosome, especially when spread over roughly the same total map length.

\section{(ii) Complete dominance, all effects unequal}

Independent genes. The probability of detecting a single dominant heterozygote is now $Q_{D \mid h}=3 / 8$, and hence the equivalent expression to (1) is

$$
P_{\text {dom }}=1-\left(1-3 / 2^{n+2}\right)^{k} \text {. }
$$

Pairs of linked genes. The probability of detecting a coupling double heterozygote,

$$
Q_{D \mid H C}=5 / 8-(1-c)^{4} / 4
$$

\begin{tabular}{|c|c|c|c|c|c|c|c|}
\hline \multicolumn{2}{|c|}{ loci } & \multicolumn{3}{|c|}{3} & \multicolumn{3}{|c|}{4} \\
\hline & $c$ & 0.2 & 0.1 & 0.05 & 0.2 & $0 \cdot 1$ & $0 \cdot 05$ \\
\hline & & 0.802 & 0.612 & 0.479 & 0.747 & 0.546 & 0.383 \\
\hline & & 0.803 & 0.640 & 0.488 & 0.765 & 0.562 & 0.436 \\
\hline & & 0.813 & 0.670 & 0.536 & 0.831 & 0.638 & 0.485 \\
\hline & & 0.831 & 0.815 & 0.565 & 0.928 & 0.687 & 0.564 \\
\hline
\end{tabular}

TABLE 2

Values of $\mathrm{d}$ for three or four loci with recombination fraction $\mathrm{c}$ between adjacent loci, unequal effects and no complete dominance (3200 Monte Carlo simulations) 
is now not equal to the probability of detecting a repulsion double heterozygote,

$$
Q_{D \mid H R}=5 / 8-c^{4} / 4 \text {. }
$$

The probability that a randomly sampled individual at generation $n$ is a coupling heterozygote, $H_{C n}$, or repulsion heterozygote $H_{R n}$, is

$$
\begin{aligned}
& H_{C n}=\frac{1}{2}\left(H_{C 1}+H_{R 1}\right)\left(\frac{1}{2}-c+c^{2}\right)^{n-1}+\frac{1}{2}\left(H_{C 1}-H_{R 1}\right)\left(\frac{1}{2}-c\right)^{n-1} \\
& H_{R n}=\frac{1}{2}\left(H_{C 1}+H_{R 1}\right)\left(\frac{1}{2}-c+c^{2}\right)^{n-1}-\frac{1}{2}\left(H_{C 1}-H_{R 1}\right)\left(\frac{1}{2}-c\right)^{n-1} .
\end{aligned}
$$

The probability of final detection is thus

$$
\mathrm{P}_{\text {dom }}=H_{C n} Q_{D \mid H C}+H_{R n} Q_{D \mid H R}+h_{n} Q_{D \mid h},
$$

which clearly depends on whether there is initially a coupling or repulsion heterozygote, $H_{C 1}=1$ or $H_{R 1}=1$, respectively.

These results can be extended to many chromosomes, as above, now using $H_{C 1}$ and $H_{R 1}$ as probabilities of coupling or repulsion heterozygotes, and a ratio $d$ equivalent to (4), obtained. Examples are given in table 3 , and include the case where coupling and repulsion heterozygotes are equally frequent initially. Notice that $d$ is initially much higher, and may exceed unity, if all heterozygotes are initially in repulsion rather than in coupling. With no recombination, the coupling case is equivalent to just a single gene, whereas in the repulsion case the heterozygote differs from both homozygotes.

\begin{tabular}{|c|c|c|c|c|c|c|c|c|c|c|}
\hline \multicolumn{5}{|c|}{$\mathbf{P}_{n}, c$} & \multicolumn{6}{|c|}{ a } \\
\hline 0.5 & \multicolumn{2}{|c|}{$0 \cdot 1$} & \multicolumn{2}{|c|}{0.01} & \multicolumn{3}{|c|}{0.1} & \multicolumn{3}{|c|}{0.01} \\
\hline$C=R$ & C & $R$ & $C$ & $R$ & $C$ & $\frac{1}{2} C \frac{1}{2} R$ & $R$ & $C$ & $\frac{1}{2} C \frac{1}{2} R$ & $R$ \\
\hline 0.6094 & 0.4610 & $0 \cdot 6250$ & 0.3849 & 0.6250 & 0.6574 & 0.8504 & $1 \cdot 0434$ & 0.5169 & 0.7802 & $1 \cdot 0434$ \\
\hline $0 \cdot 3398$ & 0.2573 & 0.3229 & $0 \cdot 1961$ & 0.3137 & 0.7164 & 0.8277 & 0.9390 & 0.5255 & 0.7160 & 0.9066 \\
\hline $0 \cdot 1787$ & 0.1396 & $0 \cdot 1658$ & 0.0998 & $0 \cdot 1575$ & 0.7636 & 0.8422 & 0.9209 & 0.5340 & 0.7022 & 0.8703 \\
\hline 0.0463 & 0.0389 & 0.0431 & $0 \cdot 0258$ & 0.0397 & 0.8370 & 0.8832 & 0.9293 & 0.5514 & 0.7022 & 0.8531 \\
\hline 0.0059 & 0.0053 & 0.0056 & 0.0034 & $0 \cdot 0050$ & 0.9094 & 0.9324 & 0.9554 & 0.5771 & 0.7164 & $0 \cdot 8558$ \\
\hline
\end{tabular}

\section{TABLE 3}

Values of $P_{\mathrm{a}}, \mathrm{c}$ and $\mathrm{d}$ (see Table 1) for complete dominance at pairs of loci with unequal effects. C denotes initial coupling, $\mathrm{R}$ initial repulsion and $\frac{1}{2} \mathrm{C}_{2} \mathrm{R}$ equal frequencies of coupling and repulsion

(iii) Equal gene effects

When the effects are the same at each locus, some genotypes can not be distinguished. With genes of equal effect and no dominance

$$
\begin{aligned}
& Q_{D \mid H C}=1-3(1-c)^{4} / 8-3 c^{2}(1-c)^{2}-c^{4}, \\
& Q_{D \mid H R}=1-3 c^{4} / 8-3 c^{2}(1-c)^{2}-(1-c)^{4} ;
\end{aligned}
$$

and with completely dominant genes of equal effect

$$
Q_{D \mid H C}=\frac{1}{2}-3(1-c)^{4} / 8+(1-c)^{2} / 4, \quad Q_{D \mid H R}=\frac{1}{2}-3 c^{4} / 8+c^{2} / 4 .
$$

These formulae can be used in (5). Values of probabilities of detection are given in table 4 for pairs of loci when there is (a) no dominance or 
(b) complete dominance. The latter case, equal effects and complete dominance, is equivalent to Jinks and Towey's $P_{\min }$. In both cases the initial state of coupling or repulsion affects the probability of detection, but in opposite directions: with no dominance the frequent segregants of repulsion heterozygotes are identical, so genes are missed, whereas with dominant genes, homozygous segregants from the repulsion heterozygote differ from the heterozygote. These results can not be generalised to several loci to compute the ratio $d$, except in the very special case where only linked pairs of genes are of equal effect. Indeed it seems unlikely that even pairs would have equal effect, assuming a near continuous distribution of effects.

\section{TABLE 4}

Values of $P_{\mathrm{n}}$, for two loci with equal effects when there is $(a)$ no dominance and $(b)$ complete dominance. $\mathrm{C}$ denotes initial coupling and $\mathrm{R}$ initial repulsion

\begin{tabular}{|c|c|c|c|c|c|}
\hline & & & $\mathbf{P}_{n}, c$ & & \\
\hline & 0.5 & & & & \\
\hline n & $C=R$ & $C$ & $R$ & C & $R$ \\
\hline & & (a) I & dominar & & \\
\hline 1 & 0.7266 & 0.7296 & 0.3196 & 0.6395 & 0.0391 \\
\hline 2 & 0.4941 & 0.4096 & 0.2456 & 0.3257 & 0.0316 \\
\hline 3 & 0.2798 & 0.2234 & $0 \cdot 1578$ & $0 \cdot 1658$ & 0.0217 \\
\hline 5 & 0.0761 & 0.0629 & 0.0528 & 0.0429 & 0.0083 \\
\hline 8 & 0.0097 & 0.0087 & 0.0080 & 0.0056 & 0.0015 \\
\hline & & (b) Con & ete domi & & \\
\hline 1 & 0.5391 & 0.4565 & 0.5025 & 0.3848 & 0.5000 \\
\hline 2 & 0.3223 & 0.2549 & 0.2733 & $0 \cdot 1960$ & 0.2525 \\
\hline 3 & $0 \cdot 1743$ & $0 \cdot 1383$ & $0 \cdot 1457$ & 0.0998 & $0 \cdot 1275$ \\
\hline 5 & 0.0461 & 0.0386 & 0.0766 & 0.0258 & 0.0325 \\
\hline 8 & 0.0058 & 0.0053 & 0.0054 & 0.0034 & 0.0042 \\
\hline
\end{tabular}

\section{Discussion}

Jinks and Towey mention the various assumptions of the genotype assay method, of which the more important will be discussed. In common with many analyses there is assumed to be no differential viability among lines and no selection; this assumption is critical in genotype assay. Whole chromosomes or long regions of chromosomes tend to be heterozygous or homozygous together in $\mathrm{F}_{n}$ individuals, so that either heterozygote superiority or simple deleterious recessives for genes unrelated to, but linked to, those affecting the quantitative trait (i.e. pseudo-overdominance) will increase the number of segregating genes and thus bias upwards estimates made in later generations. There are also problems of downward bias in that small differences in gene effects will not usually be detected by progeny testing. Jinks and Towey use a null hypothesis of no difference between each family, which is equivalent to an overall null hypothesis that there are no effective factors or that their effects are all zero. This seems unrealistic, particularly if there is any difference in performance between the constituent inbred lines. A consequence of this approach is that the number of factors detected 
changes with the scale of the experiment or the significance level in each statistical test.

The main limitation that we see in the method is that it can only estimate numbers of effective factors, and not the actual number of gene differences. The concept of effective factors is discussed extensively by Mather and Jinks (1971) and explained as follows (p. 325): "The effective factor, as we have been using it, is in the general case therefore not an ultimate gene, but merely a segment of chromosome acting as a unit of inheritance and separated from other units by an average recombination frequency of $50 \%$ ". They also add (p. 325) in a general context: "Finally, since chiasmata vary in position, a further breakdown of effective factors must occur in later generations. The total number of factors found in these later generations will generally be greater than the first estimate"

Whilst it may be possible to describe the variance in a population at some generation in terms of effective factors, they can not be used to predict the variance in generation $n+1$. For example, if an $F_{1}$ between a high and low line is estimated to have six effective factors by genotype assay using $P_{\max }$, this could be six genes each on separate chromosomes, or 10 genes each in pairs 0.05 cross-over units apart on five chromosomes (table 1). If the variance in the $F_{2}$ population were the same in each case, it would not then be so in, say, a composite $F_{3}$ population made from the $F_{2}$ lines. Similarly the rates of response would not be the same if selection were practised.

Since the effective factor is usually not a constant quantity over generations, a discussion of the optimum numbers of generations of inbreeding to estimate numbers of effective factors (Jinks and Towey, pp. 71-72) is equivocal. To estimate numbers of gene differences, many generations of inbreeding are required if the estimates are not to be biased downwards by linkage, especially for species with few chromosomes. A further disadvantage of using early generations, $F_{2}$ or $F_{3}$, is, as Jinks and Towey show in their graphs of $\mathbf{P}$ against number of effective factors (although labelled as number of loci) that the relationship is very flat with more than about five effective factors. The disadvantage, however, of using later generations is that more time is needed for the experiment, selective forces, if present, have longer to act, and the curves of $\mathrm{P}$ against number of effective factors are of low slope causing the variance in predicted number of effective factors to be high over the whole range of number of factors.

Our results show how the number of effective factors changes with generation in genotype assay, and how this depends on initial coupling/ repulsion relationships. Both the ratio of number of effective factors to number of gene differences, and the change of this ratio with generation number may be different for other methods of analysis. Indeed, as shown by (2), the degree of linkage enters into the detection of segregation in $F_{n}$ by the $F_{n+2}$ progeny test and if, for example, $F_{n}$ individuals were progeny tested directly by repeated crossing to an inbred, the factor $d$ shown in tables 1-3 would be different. Even for Nicotiana rustica, which has a large number of chromosomes, Jinks and Towey found increasing numbers of effective factors when the $\mathrm{F}_{5}$ rather than $\mathrm{F}_{3}$ was used.

Any estimate of number either of effective factors or of genes applies to the particular pair of lines used in the initial $F_{1}$ cross and can not usually be extrapolated to other lines or line combinations. If, however, the two 
lines used are extreme high and low strains from some initial population, many genes should be differentiated between them. An estimate of number and size of gene effect differences may then tell us something of the underlying biology of the trait being studied and provide a basis for modelling; the biological meaning of effective factors is less clear.

\section{RefERENCES}

JENNINGS, н. s. 1917. The numerical results of diverse systems of breeding, with respect to two pairs of characters, linked or independent, with special relation to the effects of linkage. Genetics, 2, 97-154.

JINKs, J. L., AND TOWEY, P. 1976. Estimating the number of genes in a polygenic system by genotype assay. Heredity, 37, 69-81.

Mather, K., AND Jinks, J. L. 1971. Biometrical Genetics, 2nd edition. Chapman and Hall, London. 